\title{
Effect of boundary conditions on stochastic Ising- like financial market price model
}

Wen Fang and Jun Wang ${ }^{*}$

\author{
* Correspondence: wangjun@bjtu. \\ edu.cn \\ Department of Mathematics, Key \\ Laboratory of Communication and \\ Information System, Beijing \\ Jiaotong University, 100044 Beijing, \\ P.R. China
}

\begin{abstract}
Price formation in financial markets based on the 2D stochastic Ising-like spin model is proposed, with a randomized inverse temperature of each trading day. The statistical behaviors of returns of this financial model are investigated for zero boundary condition and five different classes of mixed boundary conditions. For comparison with actual financial markets, we also analyze the statistical properties of Shanghai Stock Exchange (SSE) composite Index, Shenzhen Stock Exchange (SZSE) component Index and Hushen 300 Index. Fluctuation properties, fat-tail phenomena, power-law distributions and fractal behaviors of returns for these indexes and the simulative data are studied. With the plus boundary condition, for example the boundary condition $i \sigma$, the value of market depth parameter $i$ is smaller than those of the corresponding market depth parameters $i$ with zero boundary condition i1 and weak mixed boundary conditions iz and iz. And the changing range of tails exponents of boundary condition $i_{6}$ is much smaller than those of the other five boundary conditions.
\end{abstract}

Keywords: stochastic Ising-like spin model, boundary condition, financial time series, statistical analysis, stock market

\section{Introduction}

As the stock markets are becoming deregulated worldwide, the modeling of the dynamics of the forward prices is becoming a key problem in risk management, physical assets valuation and derivatives pricing, see [1-6], and it is also important to understand the statistical properties of fluctuations of stock price in globalized securities markets, for example see $[7,8]$. A complex behavior can emerge due to the interactions among smallest components of that system, see [9], and it is often a successful strategy to analyze the behavior of a complex system by studying these components. In financial markets, these components are comprised by the market participants who buy and sell assets in order to realize their trading and investment decisions. Similar to physical systems, the superimposed flow of all individual orders submitted to the exchange trading system initiated by market participants and its change in time generate a complex system with fascinating properties, see [1,2].

Recently, the theory of stochastic interacting particle systems [10-12] has been applied to investigate the statistical behaviors of fluctuations for stock prices, and the corresponding valuation and hedging of contingent claims for these price process models are also studied, see $[1,2,11,12]$. In the present article, we suppose that traders

\section{Springer}

(c) 2012 Fang and Wang; licensee Springer. This is an Open Access article distributed under the terms of the Creative Commons Attribution License (http://creativecommons.org/licenses/by/2.0), which permits unrestricted use, distribution, and reproduction in any medium, provided the original work is properly cited. 
determine their positions at each time by observing the market information (and then evaluating the market behavior, market sentiment and their trading strategies), each trader is thought to be a subunit in the stock market, and may take positive (buying) position, negative (selling) position or neutral position, denoted by,+- , and 0 , respectively. Traders with buying positions or selling positions are called market participants, and the configuration of positions for all traders is assumed as the main factor resulting in price fluctuations in this financial model. The reason that we use interacting particle systems to investigate the fluctuation of stock markets is that all of these systems consist of subunits. The Ising spin system, which can describe the mechanism of making a decision in a closed community, is the most popular ferromagnetic model of interacting particle systems. The subunits in a 2D Ising model are called spins (with the interactions between the nearest neighbors), the clusters of parallel spins in the square-lattice Ising model can be defined as groups of traders acting together on the stock market model, for example see $[1,3,4]$. The objective of this work is to study the financial phenomena of the price model developed by the stochastic Ising-like spin model. In this model, all of the spins are flipped by following Ising dynamic system [13], and the inverse temperature of each trading day is randomly chosen in a certain interval. And for different boundary conditions, the statistical behaviors of the price model are studied. Further, the empirical research in financial market fluctuations for the actual stock market and the financial model is made by comparison analysis.

\section{Description of 2D Ising-like spin model}

Considering the Ising model on 2D integer lattice $i^{2}$, at sufficiently low temperatures, we have known that the model exhibits phase transition, i.e., there is a critical point $\beta_{\mathrm{c}}$ $>0$, if $\beta>\beta_{c}$, the Ising model exhibits the phase transition, for more details see $[4,14,15]$. Let $i^{2}$ be the usual 2D square lattice with sites $u=\left(u_{1}, u_{2}\right)$, equipped with the $l_{1}$-norm: ||$u||=\left|u_{1}\right|+\left|u_{2}\right|$. Given $i \dot{i} i^{2}$ and $i^{c}=i^{2}-i, i_{i}=\{-1,+1\}^{i}$ is the configuration space. An element of $i_{i}=\{-1,+1\}^{i}$ will usually denote by $i_{i}=\{i(u): u i$ i). Whenever confusion does not arise, we will also omit the subscript $i$ in the notation $\dot{\varepsilon}_{i}$, and we also denote by $\left.\right|_{i} \mid$ the cardinality of $\dot{i}$. The set $B_{\dot{i}}$ of bonds in $\dot{i}$ is defined by $B_{i}=\{(u, v) \quad i \quad \times i:\|u-v\|=1\}$, which means the bonds of vertical and horizontal nearest neighbors but not diagonal neighbors. Given a boundary condition $\tau \in \Omega_{\mathbb{Z}^{2}}=\{-1,0,+1\}^{\mathbb{Z}^{2}}$, we consider the Hamiltonian

$$
H_{\Lambda}^{\tau}(\omega)=-\sum_{(u, v) \in B_{\Lambda}} \omega(u) \omega(v)-\sum_{\substack{(u, v) \in \Lambda \times \Lambda^{c} \\\|u-v\|=1}} \omega(u) \tau(v) .
$$

The Gibbs measure associated with the Hamiltonian is defined as

$$
\mu_{\Lambda}^{\beta, \tau}(\omega)=\exp \left[-\beta H_{\Lambda}^{\tau}(\omega)\right] / \sum_{\omega \in \Omega_{\Lambda}} \exp \left[-\beta H_{\Lambda}^{\tau}(\omega)\right]
$$

where $\beta>0$ is a parameter. The stochastic dynamics that we want to study is defined by the Markov generator

$$
\left(\mathcal{A}_{\Lambda}^{\beta, \tau} f\right)(\omega)=\sum_{u \in \Lambda} c(u, \omega, \tau)\left[f\left(\omega^{u}\right)-f(\omega)\right]
$$


acting on $L^{2}\left(\Omega_{\Lambda}, d \mu_{\Lambda}^{\beta, \tau}\right)$, where $\dot{i}^{u}(v)=+\dot{i}(v)$ if $v i u$, and $\dot{i}^{u}(v)=-\dot{i}(v)$, if $v=u . c(u$, $\dot{i}, \dot{i})$ is the transition rates for the process [15], satisfying nearest neighbor interactions, attractivity, boundedness and detailed balance condition $c(u, \omega, \tau) \mu_{\Lambda}^{\beta, \tau}(\omega)=c\left(u, \omega^{u}, \tau\right) \mu_{\Lambda}^{\beta, \tau}\left(\omega^{u}\right)$. In the present article, we take

$$
c(u, \omega, \tau)=\exp \left\{-\beta \omega(u)\left[\sum_{v \in \Lambda,\|u, v\|=1} \omega(v)+\sum_{(u, v) \in \partial \Lambda} \tau(v)\right]\right\}
$$

where we define the interior and exterior boundaries of $i$ as

$$
\partial_{\text {int }} \Lambda=\{u \in \Lambda: \exists v \notin \Lambda,\|u-v\|=1\}, \quad \partial_{\text {ext }} \Lambda \equiv\{u \notin \Lambda: \exists v \in \Lambda,\|u-v\|=1\}
$$

and the edge boundary $i i$ as

$$
\partial \Lambda=\left\{(u, v): u \in \partial_{\text {int }} \Lambda, v \in \partial_{\text {ext }} \Lambda,\|u-v\|=1\right\} .
$$

If we set $\dot{i}(u)=0$ for all $u \dot{i} \dot{i}^{2}$, then we call the resulting boundary condition the zero or open boundary condition, if $\dot{i}(u)=+1$ for all $u i_{i} i^{2}$, the boundary condition is called the plus boundary condition, if $\dot{i}(u)=-1$ for all $u \dot{i} \dot{i}^{2}$, then the resulting boundary condition is called the minus boundary condition, and if there are either $\dot{i}(u)=+1$ or $\dot{i}(u)=-1$ for some $u \dot{i} \dot{i}^{2}$, and $\dot{i}(u)=0$ for the others, we call this kind of condition the mixed boundary condition. The spin of the Ising model can point up (spin value +1 ) or point down (spin value -1), and it flips between the two orientations. At sufficiently low temperatures, the energy effect predominates and we have known that the model exhibits phase transition. Correlations are related to the phase transition and the spin fluctuations of the model. As $\beta$ increases (from 0 ), the correlations begin to extend, these correlations take the form of spin fluctuations, which are islands of a few spins each that mostly point in the same direction. As $\beta$ approaches the critical inverse temperature $\beta_{c}$ from below, spin fluctuation are present at all scales of length. At $\beta=$ $\beta_{c}$, the correlations decay by a power law, but for $\beta>\beta_{c}$, there are two distinct pure phases. Correlations play an important role in studying the fluctuations of the phase interfaces for the statistical physics model, see $[4,14]$. In the following section, since the financial price model heavily depends on the number of spin values, we set the intensity of interaction among the market investors $\beta=i \dot{i}$, where $i$ is a random variable with the uniform distribution in $0[1]$, and $i$ is a intensity parameter, then we obtain the Ising-like spin model.

\section{Financial model and boundary conditions}

In this section, we develop a financial price model by Ising-like spin dynamic system. For a stock market, we consider a single stock and assume that there are $n^{2}$ traders in this stock, and each trader can trade unit number of stocks at each time $t$. At each time $t$, the behavior of stock price process is determined by the number of traders $x$ ${ }^{+}(t)$ (with buying positions) and $x^{-}(t)$ (with selling positions). If the number of traders in buying positions is larger than that of traders in selling positions, it implies that the stock price is considered to be low by the market participants, and the stock price auctions higher searching for buyers, similarly for the opposite case. Let $x_{i j}(t)$ be the investing position of a trader $\left(1_{i} i_{i} n, 1_{i} j i n\right)$ at time $t$, and $x(t)=\left(x_{11}(t), \ldots, x_{1 n}\right.$ $\left.(t), \ldots, x_{n 1}(t), \ldots, x_{n n}(t)\right)$ be the configuration of positions for $n^{2}$ traders. A space of all 
configurations of positions for $n^{2}$ traders from time 1 to $t$ is given by $\mathcal{X}=\{x=(x(1), \ldots, x(t))\}$. For a given configuration $x \in \mathcal{X}$ and a trading day $t$, let

$$
\mathcal{N}(x(t))=x^{+}(t)-x^{-}(t)
$$

Suppose that $i t(x)$ is a random variable which represents the information arrived on the th trading day, where $i t=1$ for buying positions, $i t=-1$ for selling positions and $i t=0$ for neutral positions with probability $p_{1}, p_{-1}$, and $1-\left(p_{1}+p_{-1}\right)$ respectively. Then these investors send bullish, bearish or neutral signal to the market. If $\xi_{t}(x)|\mathcal{N}(x(t))|>0$, there are more buyers than sellers, then the stock price is auctioned up, similarly for other cases. From the above definitions and $[2,4,5]$, we define the stock price of the model at time $t(t=1,2, \ldots)$ as $S_{t}=e^{\gamma \xi_{t}(x)|\mathcal{N}(x(t))| / n^{2}} S_{t-1}$, where $i>$ 0 is the depth parameter of the market, and $S_{0}$ be the the initial price at time $t=0$. Then, we have

$$
S_{t}=S_{0} \exp \left\{\gamma \sum_{k=1}^{t} \frac{\xi_{k}(x)|\mathcal{N}(x(k))|}{n^{2}}\right\} .
$$

The formula of the single-period stock logarithmic returns from $t-1$ to $t$ is given by

$$
r(t)=\ln S_{t}-\ln S_{t-1} .
$$

Next, we consider the different boundary conditions for the model in a finite square $i$, which is defined by $i=\left\{\left(u_{1}, u_{2}\right): 1 \dot{i} u_{1} i n, 1 i u_{2} i n\right\}$ for a large integer $n$, and

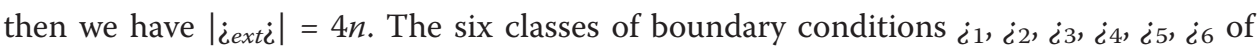
the financial model based on the Ising-like system are given as follows, where $\tau_{i} \in\{+1,0,-1\}^{\mathbb{Z}^{2}}$ for $i=1, \ldots, 6$.

A. Boundary Condition 1. The boundary condition $i 1$ is defined as follows: $i 1(u)=0$ for all $u \dot{i} \dot{i} e x t \dot{i} \cdot \dot{\Sigma}_{1}(u)=0$ means that the site $u$ is open or there is no spin on the site $u$, this boundary condition is called the zero boundary condition.

B. Boundary Condition 2. The boundary condition $i_{2}$ is defined as follows: Starting from the site $(1, n+1)$, we give a clockwise order to the sites in $i$ ext $i$, that is $\left\{u^{i}, i=1, \ldots\right.$, $4 n\}$. Assume that there are two positive integers $l$ and $m$ such that $4 n=l m$ for a properly chosen large integer $n$. For any $u^{i} i$ iext $i, i=1, \ldots, 4 n$, we set

$$
\tau_{2}\left(u^{i}\right)=\left\{\begin{array}{l}
+1, \quad l m_{1}<i \leq l\left(m_{1}+1\right), \text { for some } m_{1}=0,2,4,6, \ldots \\
-1, \quad \text { otherwise }
\end{array}\right.
$$

where $m_{1}$ is a positive integer which depends on the number $4 n$ for a fixed $l$. In this case, for the first connected sites of length $l$ in $\left\{u^{i}\right\}$ from the site $(1, n+1)$, we assign the same spins (" + "spin) on these sites, then on the next connected sites of length $l$ we assign the " - " spins on these sites, and so on. This is a mixed boundary condition, and suppose that $l=10$ in the following parts of the present article.

C. Boundary Condition 3. The boundary condition $i z$ is defined as follows: For any $u$ $=\left(u_{1}, u_{2}\right) i i^{2}$, let

$$
\tau_{3}(u)=\left\{\begin{array}{ll}
+1, & \text { if } u_{2} \geq n+1 \text { or } u_{2} \leq 0 \\
-1, & \text { otherwise }
\end{array} .\right.
$$


In this case, the plus spins are assigned on the top side and the bottom side of the exterior boundary $i_{\text {ext }} \dot{i}$, and the minus spins are assigned on the left side and the right side of iexti.

D. Boundary Condition 4. The boundary condition $\dot{\iota}_{4}$ is defined as follows: Suppose that the boundaries of the top side, the bottom side and the left side of the exterior boundary iexti are zero boundary conditions, and the right side of $i$ ext $i$ is defined by

$$
\tau_{4}\left(n+1, u_{2}\right)= \begin{cases}-1, & \text { if } u_{2}=3 m_{2}, m_{2}=1,2, \ldots \\ +1, & \text { otherwise }\end{cases}
$$

where $m_{2}$ is a positive integer such that $m_{2}<n / 3$.

E. Boundary Condition 5. The boundary condition $i_{5}$ is defined as follows: Four sides boundary conditions of the exterior boundary iexti are same as that of the right side boundary condition in $i 4$.

F. Boundary Condition 6. The boundary condition $\dot{i 6}$ is defined as follows: $\dot{\iota}(u)=+1$ for all $u i i^{2}$, the boundary condition is called the plus boundary condition.

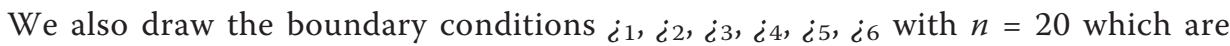
described in Figure 1. For the above boundary conditions $i_{1}, i_{2}$ and $i_{3}$, neither " + " nor " - " predominates the other, whereas the overwhelming part of the boundary sites in $\dot{i}_{4}, i_{5}$ and $i_{6}$ is plus. In a stock market, here the boundary condition $\dot{i}$ may represent the information or the situation on this stock, including the estimation for this stock price, positive or negative news, trends, political event and economic policy, etc.

In our computer simulations, the system size is $n=100$, and the position probabilities $p_{1}=p_{-1}=0.5$. Each step represents one trading minute, 240 steps constitute one trading day, the random variable $\dot{i}$ is stochastically chosen in the uniform distribution

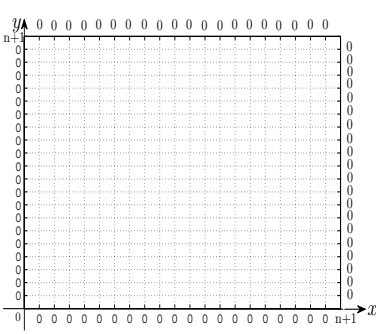

(a)



(d)



(b)

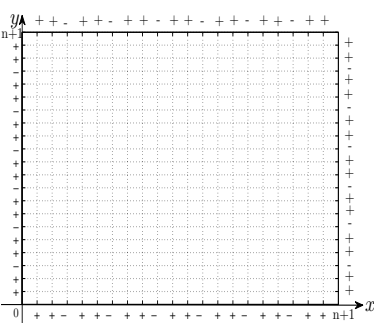

(e)

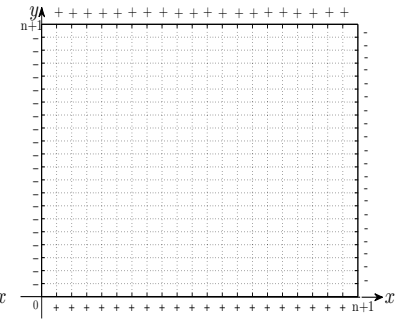

(c)

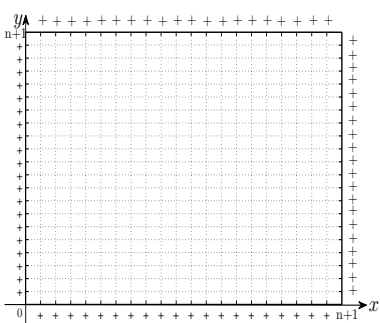

(f)

Figure 1 The plots of six classes of the boundary conditions. (a-f) The six classes of boundary conditions $i_{1}, i_{2}, i_{3}, i_{4}, i_{5}$, and $i_{6}$ with $n=20$ respectively. 
$0[1]$ once for every step, where the value of inverse temperature $\beta=i i$ is defined in Section 2. We typically simulate 1200000 steps, which corresponds to 5000 trading days for each boundary condition and each fixed intensity parameter $i$. We analyze the historical data sets of Hushen 300 Index, Shanghai Stock Exchange (SSE) Composite Index and Shenzhen Stock Exchange (SZSE) Component Index, which records every trade for all the securities in the Chinese stock market during the period from January 4, 2005 to December 31, 2010, a total number of observed trading days is 1457, see http://www.sse.com.cn and http://www.szse.cn. The Hushen 300 Index consists of 300 actively traded large cap companies in the SSE (179 companies) and SZSE (121 companies), which has a good representative of the market. For these databases, the records of the daily closing price are continuous in regular open days for every week, due to the removal of all market closure times.

According to the definitions of the logarithmic changes of stock price from the $t$ 1 th day to th day in (1) and (2), we plot the figures of the stock price series and the returns by simulating the financial model with the parameter $i=0.8$ in the zero boundary condition $\dot{\iota}_{1}$ and the Hushen 300 Index, see Figure 2a-d. We also plot the probability density functions (PDF) of SZSE Component Index and the financial model with the zero boundary condition $\dot{i 1}$ for different parameter values $i$, and the corresponding Gaussian distribution is plotted for comparison in Figure 3. Comparing with the Gaussian distribution, the probability densities of SZSE Component Index and the simulative data with the zero boundary conditions $i 1$ obviously show the phenomena of the peak distributions in Figure 3. And when the value $i$ increases, the peak phenomenon of the returns are more evidently. The peak distribution of simulative data with $i_{1}$ and $i=0.5$ is much closer to that of SZSE Component Index.
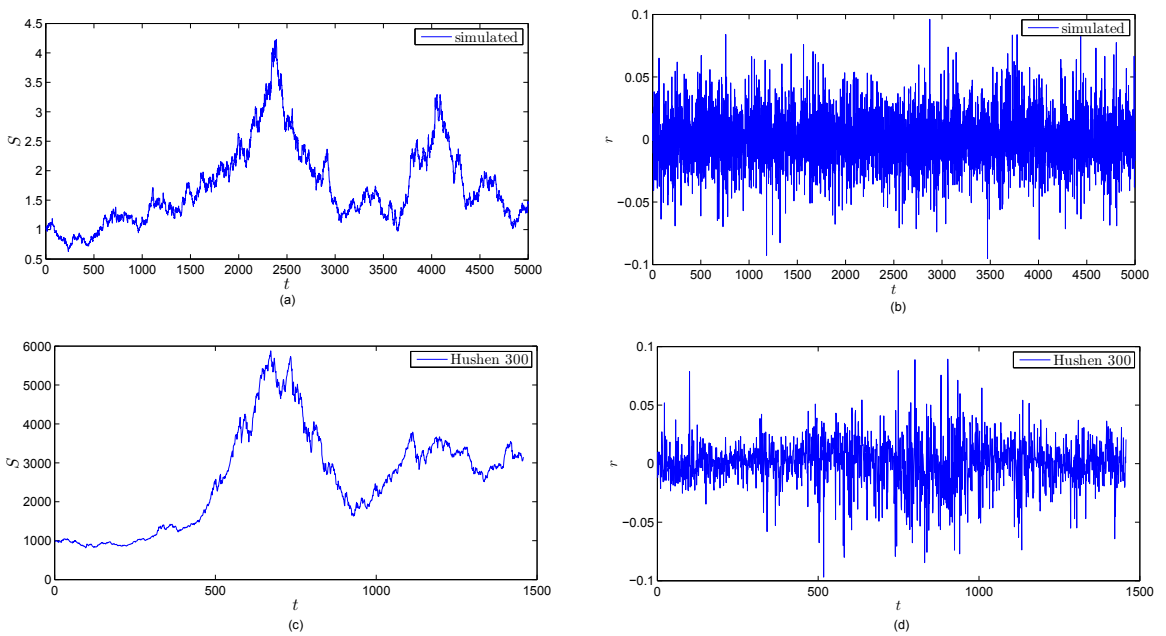

Figure 2 The fluctuations of stock prices and the corresponding returns. (a) The price time series simulated by the price model with the zero boundary condition $i_{1}$ for $i=0.8, i=0.58$. (b) The corresponding logarithmic returns of the simulated price time series. (c) The closing prices of Hushen 300 Index from January 4, 2005 to December 31, 2010. (d) The corresponding logarithmic returns of Hushen 300 Index 




Figure 3 The probability density function (PDF) of logarithmic returns of SZSE Component Index and the price model with the zero boundary condition $i_{1}$ for different intensity values $i=1.0, i=$ $0.8, i=0.6, i=0.5, i=0.45, i=0.4$ and $i=0.3$. And the corresponding Gaussian distribution is plotted for comparison.

\section{Statistical behaviors of financial model with different boundary conditions}

Statistical behaviors of price fluctuations are very important to understand and model financial market dynamics, which have long been a focus of economic research. Stock price volatility is of interest to traders because it quantifies risk, optimizes the portfolio, and provides a key input of option pricing models that are based on the estimation of the volatility of the asset, see [6,7].

\subsection{The statistical properties of the model}

In this section, we investigate the statistical properties of the financial model with six classes boundary conditions, including the kurtosis and the skewness of the market returns. Kurtosis is a measure of the flatness of the probability distribution for a real valued random variable. Higher kurtosis which means more of the variance is due to infrequent extreme deviations, as opposed to frequent modestly sized deviations. It is known that the kurtosis of the Gaussian distribution is 3, while the kurtosis of the real markets is usually larger than 3 by the empirical research. The recent research shows that returns on the financial markets are not Gaussian, but exhibit the excess kurtosis and the fatter tails than the normal distribution, which is usually called the "fat-tail" phenomenon, see [2]. The kurtosis and skewness are defined as follows

$$
\text { kurtosis }=\frac{\sum_{t=1}^{n}\left(r_{t}-\bar{r}\right)^{4}}{(n-1) \sigma^{4}}, \quad \text { skewness }=\frac{\sum_{t=1}^{n}\left(r_{t}-\bar{r}\right)^{3}}{(n-1) \sigma^{3}}
$$

where $r_{t}$ denotes the return of $t$ th trading day, $\bar{r}$ is the mean of $r, n$ is the total number of the data, and $\dot{i}$ is the corresponding standard variance. The kurtosis shows the centrality of data and the skewness shows the symmetry of the data. It is known that the skewness of standard normal distribution is 0 .

In Table 1, we consider the statistics of returns for SSE Composite Index, SZSE Component Index and Hushen 300 Index from January 4, 2005 to December 31, 2010. This shows that three kurtosis vlaues of returns of the Chinese stock market indexes 
Table 1 The statistical properties of Chinese stock market

\begin{tabular}{lllllll}
\hline & Mean & Variance & Max & Min & Kurtosis & Skewness \\
\hline SSE & 0.000546 & 0.000382 & 0.090345 & -0.092561 & 5.584019 & -0.347366 \\
SZSE & 0.000962 & 0.000466 & 0.091615 & -0.097501 & 4.962429 & -0.377435 \\
Hushen 300 & 0.000786 & 0.000431 & 0.089310 & -0.096949 & 5.267240 & -0.412498 \\
\hline
\end{tabular}

are larger than 3, which implies that these returns exhibits the excess kurtosis and the fatter tails than the corresponding Gaussian distributions, and the biased distributions of skewness also exist for the indexes. Since the range of daily price fluctuation is limited in Chinese stock markets, that is, the changing limits of daily returns for stock prices and stock market indexes are between $-10 \%$ and $10 \%$, then the value of market depth parameter $i$ (which is defined in (1) of Section 3) depends on the given intensity value of $\dot{i}$, in an attempt to make the financial price model satisfy the changing limits of daily returns for Chinese stock markets. In the simulation of the financial model, the statistical properties of the returns for different boundary conditions are displayed in Table 2. They show that the kurtosis values are also larger than 3, and the kurtosis value is becoming smaller with the intensity $i$ decreasing for each boundary condition.

In Table 2 , for each fixed boundary condition $i$, as the intensity parameter $\dot{i}$ decreases, the depth parameter $\dot{i}$ of the model has the tend to increase. The interaction among the market investors decreases for the intensity parameter decreasing, this means that investors may pay less attention to the other people's investment attitude around them. In this situation, Table 2 shows that the market depth parameter may play a great role in the price model. And for each fixed value of intensity parameter $i$, when the boundary conditions are neither " + " nor " - " predominates the other, for example the boundary conditions $i_{1}, \dot{\varepsilon}_{2}$, and $\dot{i}_{3}$, the values of depth parameters $\dot{i}$ are larger than that of the corresponding depth parameter $i$ of plus boundary condition $i 6$, which is the plus is the overwhelming part of the boundary sites. When the interaction among the market investors increases or the external environment is dominant by one view for a long time, the value of market depth parameter $i$ may decrease. And the ranges of variances of returns with six boundary conditions $\dot{i 1}, \dot{i 2}, \dot{i} 3, \dot{i} 4, \dot{i} 5, \dot{i} 6$ are [0.00037, 0.00068], [0.00031, 0.00067], [0.00045, 0.00067], [0.00033, 0.00067], [0.00033, 0.00050], [0.00037, 0.00057], respectively. Since the variances of SSE, SZSE and Hushen 300 are $0.000382,0.00046$, and 0.000431 , respectively, from the view of fluctuations, the simulation of model with boundary condition $i 5$ is closer to this period of January 4, 2005 to December 31, 2010 in real Chinese stock markets.

For understanding the difference between the normal distribution and the distributions of SSE Index, SZSE Index, Hushen 300 Index and the simulative data, we also make single-sample Kolmogorov-Smirnov test [16] by the statistical method. We compute the statistical values of the returns for Hushen 300 Index, SSE Index, SZSE Index, and the simulative data numerically, see Tables 3 and 4 after normalizing these time series. The null hypothesis is that the data vector has a standard normal distribution. The alternative hypothesis is that the the sample does not have that distribution. The result $h$ is 1 if the test rejects the null hypothesis at the $5 \%$ significance level, 0 otherwise. The $p$-value $p$, the test statistic $k$, and the cutoff value $c v$ for determining whether $k-s$ statistical is significant. From Tables 3 and 4, they indicate that the behaviors of the financial model are close to the real market when the intensity $i$ ranges from 0.4 to 
Table 2 The statistical properties of price model with six classes boundary conditions

\begin{tabular}{|c|c|c|c|c|c|c|c|c|}
\hline Boundary & $i$ & $i$ & Mean & Variance & Max & Min & Kurtosis & Skewness \\
\hline$i_{1}$ & 1 & 0.5 & 0.00003 & 0.00040 & 0.09890 & -0.09380 & 4.85178 & 0.13589 \\
\hline$i_{1}$ & 0.8 & 0.58 & 0.00007 & 0.00037 & 0.09616 & -0.09512 & 4.88207 & 0.10954 \\
\hline$i_{1}$ & 0.6 & 0.9 & -0.00043 & 0.00050 & 0.09792 & -0.08802 & 4.46219 & 0.13983 \\
\hline ¿1 & 0.5 & 0.92 & 0.00009 & 0.00039 & 0.09090 & -0.09734 & 4.35889 & -0.08853 \\
\hline$i_{1}$ & 0.45 & 1.1 & -0.00028 & 0.00048 & 0.09680 & -0.09416 & 4.17186 & -0.07398 \\
\hline$i_{1}$ & 0.4 & 1.5 & 0.00034 & 0.00068 & 0.09480 & -0.09810 & 3.49030 & -0.02603 \\
\hline$i_{1}$ & 0.3 & 1.7 & 0.00048 & 0.00063 & 0.09554 & -0.09656 & 3.37645 & 0.05148 \\
\hline i2 & 1 & 0.5 & 0.00059 & 0.00044 & 0.09662 & -0.09870 & 4.79473 & 0.05917 \\
\hline$i_{2}$ & 0.8 & 0.59 & 0.00019 & 0.00037 & 0.09853 & -0.08767 & 5.10466 & 0.06324 \\
\hline ¿2 & 0.6 & 0.7 & 0.00037 & 0.00031 & 0.09982 & -0.09100 & 4.93810 & 0.07865 \\
\hline$i 2$ & 0.5 & 1.05 & 0.00049 & 0.00048 & 0.09303 & -0.09618 & 4.04856 & 0.06254 \\
\hline$i 2$ & 0.45 & 1.1 & 0.00028 & 0.00046 & 0.09812 & -0.09108 & 4.11700 & 0.10904 \\
\hline$i 2$ & 0.4 & 1.2 & 0.00020 & 0.00045 & 0.09552 & -0.09888 & 3.85836 & -0.02008 \\
\hline i2 & 0.3 & 1.75 & 0.00004 & 0.00067 & 0.09135 & -0.09835 & 3.32317 & -0.00956 \\
\hline i3 & 1 & 0.53 & 0.00058 & 0.00045 & 0.09932 & -0.09158 & 5.00763 & 0.12602 \\
\hline i3 & 0.8 & 0.66 & 0.00035 & 0.00048 & 0.09332 & -0.09913 & 4.61243 & -0.00751 \\
\hline i3 & 0.6 & 0.85 & 0.00010 & 0.00045 & 0.09571 & -0.09758 & 4.69311 & 0.00167 \\
\hline i3 & 0.5 & 1.05 & 0.00033 & 0.00047 & 0.09744 & -0.09891 & 4.11189 & 0.00977 \\
\hline i3 & 0.45 & 1.1 & 0.00048 & 0.00053 & 0.09724 & -0.09856 & 4.12251 & 0.04151 \\
\hline i3 & 0.4 & 1.25 & 0.00024 & 0.00049 & 0.09875 & -0.09425 & 3.79751 & -0.00912 \\
\hline is & 0.3 & 1.75 & 0.00007 & 0.00067 & 0.09135 & -0.09975 & 3.32325 & -0.01994 \\
\hline$i_{4}$ & 1 & 0.48 & -0.00004 & 0.00039 & 0.09504 & -0.09926 & 4.76332 & -0.00688 \\
\hline$i_{4}$ & 0.8 & 0.64 & 0.00035 & 0.00043 & 0.09894 & -0.09536 & 4.93876 & 0.13699 \\
\hline$i_{4}$ & 0.6 & 0.7 & 0.00037 & 0.00033 & 0.09688 & -0.09898 & 5.14323 & 0.09849 \\
\hline$i_{4}$ & 0.5 & 0.85 & 0.00019 & 0.00033 & 0.09367 & -0.09979 & 4.49162 & -0.04789 \\
\hline$i_{4}$ & 0.45 & 1.2 & -0.00022 & 0.00057 & 0.09216 & -0.09648 & 3.84336 & -0.03486 \\
\hline$i_{4}$ & 0.4 & 1.3 & 0.00014 & 0.00053 & 0.09178 & -0.09880 & 3.87194 & -0.03044 \\
\hline$i_{4}$ & 0.3 & 1.75 & 0.00027 & 0.00067 & 0.09240 & -0.09765 & 3.35798 & 0.02708 \\
\hline$i_{5}$ & 1 & 0.41 & 0.00010 & 0.00037 & 0.09938 & -0.09479 & 4.92090 & 0.09551 \\
\hline is & 0.8 & 0.46 & 0.00030 & 0.00033 & 0.09752 & -0.09504 & 5.85585 & -0.16673 \\
\hline$i_{5}$ & 0.6 & 0.73 & 0.00031 & 0.00041 & 0.09928 & -0.09505 & 4.73252 & 0.16074 \\
\hline is & 0.5 & 0.88 & -0.00036 & 0.00042 & 0.09979 & -0.09258 & 4.88828 & 0.01417 \\
\hline is & 0.45 & 0.95 & 0.00016 & 0.00040 & 0.09937 & -0.09804 & 4.43247 & -0.10505 \\
\hline$i_{5}$ & 0.4 & 1.2 & 0.00052 & 0.00052 & 0.09888 & -0.09264 & 3.99503 & 0.05671 \\
\hline$i_{5}$ & 0.3 & 1.42 & -0.00023 & 0.00050 & 0.09883 & -0.09656 & 3.56444 & 0.00311 \\
\hline i6 & 1 & 0.3 & 0.00021 & 0.00037 & 0.09951 & -0.09877 & 4.62543 & 0.14243 \\
\hline$i_{6}$ & 0.8 & 0.48 & -0.00025 & 0.00057 & 0.09168 & -0.09936 & 4.38937 & -0.09951 \\
\hline i6 & 0.6 & 0.55 & 0.00053 & 0.00038 & 0.09680 & -0.09768 & 4.62340 & 0.08621 \\
\hline i6 & 0.5 & 0.78 & -0.00011 & 0.00051 & 0.09625 & -0.09937 & 4.14862 & -0.00981 \\
\hline ¿6 & 0.45 & 0.84 & 0.00038 & 0.00046 & 0.09912 & -0.09442 & 4.14778 & 0.01051 \\
\hline i6 & 0.4 & 0.92 & -0.00006 & 0.00042 & 0.08777 & -0.09954 & 3.97269 & 0.07773 \\
\hline$i_{6}$ & 0.3 & 1.3 & 0.00014 & 0.00051 & 0.09074 & -0.09698 & 3.81253 & -0.07299 \\
\hline
\end{tabular}

Table 3 Power law and fractal behavior of Chinese stock markets

\begin{tabular}{llllllll}
\hline Stock & $\boldsymbol{i}$ & $\boldsymbol{H}_{\mathbf{1}}$ & $\boldsymbol{H}_{\mathbf{2}}$ & $\boldsymbol{h}$ & $\boldsymbol{p}$ & $\boldsymbol{c v}$ & $\boldsymbol{k}$ \\
\hline Hushen 300 & 2.9755 & 0.69374 & 0.64784 & 1 & $3.89 \mathrm{E}-06$ & 0.0355 & 0.067 \\
SSE & 2.9291 & 0.69399 & 0.6505 & 1 & $2.17 \mathrm{E}-08$ & 0.0355 & 0.0791 \\
SZSE & 3.0819 & 0.70723 & 0.6445 & 1 & $1.17 \mathrm{E}-05$ & 0.0355 & 0.0641 \\
\hline
\end{tabular}


Table 4 The power law and fractal behavior of the financial price model

\begin{tabular}{|c|c|c|c|c|c|c|c|c|}
\hline Boundary & $i$ & $i$ & $H_{1}$ & $\mathrm{H}_{2}$ & $h$ & $p$ & $c v$ & $k$ \\
\hline$i_{1}$ & 1 & 2.9858 & 0.62932 & 0.52649 & 1 & $3.52 \mathrm{E}-14$ & 0.0192 & 0.0562 \\
\hline$i_{1}$ & 0.8 & 2.9914 & 0.62918 & 0.56231 & 1 & $1.28 \mathrm{E}-13$ & 0.0192 & 0.0551 \\
\hline$i_{1}$ & 0.6 & 3.1079 & 0.62977 & 0.54157 & 1 & 1.50E-10 & 0.0192 & 0.0482 \\
\hline$i_{1}$ & 0.5 & 3.1914 & 0.62464 & 0.57616 & 1 & 3.48E-05 & 0.0192 & 0.0331 \\
\hline$i_{1}$ & 0.45 & 3.2673 & 0.60693 & 0.49562 & 1 & 1.93E-05 & 0.0192 & 0.0339 \\
\hline$i_{1}$ & 0.4 & 3.5877 & 0.61531 & 0.52876 & 1 & 0.0242 & 0.0192 & 0.021 \\
\hline$i_{1}$ & 0.3 & 3.7026 & 0.62881 & 0.54277 & 0 & 0.0671 & 0.0192 & 0.0184 \\
\hline i2 & 1 & 2.9605 & 0.65835 & 0.57159 & 1 & 2.99E-14 & 0.0192 & 0.0564 \\
\hline$i 2$ & 0.8 & 2.9962 & 0.64852 & 0.57482 & 1 & 7.42E-13 & 0.0192 & 0.0534 \\
\hline i2 & 0.6 & 3.0368 & 0.60431 & 0.57264 & 1 & 8.08E-09 & 0.0192 & 0.0439 \\
\hline$i 2$ & 0.5 & 3.2509 & 0.61399 & 0.50458 & 1 & $1.62 \mathrm{E}-06$ & 0.0192 & 0.0374 \\
\hline i2 & 0.45 & 3.2829 & 0.62111 & 0.55096 & 1 & 2.45E-05 & 0.0192 & 0.0336 \\
\hline$i 2$ & 0.4 & 3.4894 & 0.65298 & 0.54266 & 1 & $3.58 \mathrm{E}-04$ & 0.0192 & 0.0293 \\
\hline$i 2$ & 0.3 & 3.7573 & 0.62058 & 0.512 & 0 & 0.0584 & 0.0192 & 0.0188 \\
\hline i3 & 1 & 2.9426 & 0.62758 & 0.52255 & 1 & $2.33 \mathrm{E}-14$ & 0.0192 & 0.0566 \\
\hline i3 & 0.8 & 3.1303 & 0.64643 & 0.58522 & 1 & 1.95E-12 & 0.0192 & 0.0525 \\
\hline i3 & 0.6 & 3.0115 & 0.64581 & 0.5978 & 1 & 4.02E-09 & 0.0192 & 0.0447 \\
\hline i3 & 0.5 & 3.3363 & 0.60074 & 0.52125 & 1 & 5.80E-06 & 0.0192 & 0.0357 \\
\hline$i 3$ & 0.45 & 3.235 & 0.60871 & 0.48946 & 1 & 2.68E-05 & 0.0192 & 0.0335 \\
\hline i3 & 0.4 & 3.4714 & 0.65577 & 0.54144 & 1 & $6.15 \mathrm{E}-04$ & 0.0192 & 0.0284 \\
\hline i3 & 0.3 & 3.7332 & 0.61767 & 0.49896 & 0 & 0.1043 & 0.0192 & 0.0172 \\
\hline$i_{4}$ & 1 & 3.0509 & 0.63533 & 0.57367 & 1 & 1.33E-13 & 0.0192 & 0.055 \\
\hline$i_{4}$ & 0.8 & 2.9779 & 0.64633 & 0.53821 & 1 & 4.68E-12 & 0.0192 & 0.0517 \\
\hline$i_{4}$ & 0.6 & 2.9488 & 0.63554 & 0.54294 & 1 & 2.68E-12 & 0.0192 & 0.0522 \\
\hline$i_{4}$ & 0.5 & 3.2594 & 0.6237 & 0.53025 & 1 & $1.61 \mathrm{E}-05$ & 0.0192 & 0.0342 \\
\hline$i_{4}$ & 0.45 & 3.4414 & 0.60189 & 0.46971 & 1 & 1.88E-04 & 0.0192 & 0.0304 \\
\hline$i_{4}$ & 0.4 & 3.433 & 0.62462 & 0.57237 & 1 & 0.002 & 0.0192 & 0.0263 \\
\hline$i_{4}$ & 0.3 & 3.7723 & 0.63278 & 0.54339 & 0 & 0.0528 & 0.0192 & 0.019 \\
\hline$i_{5}$ & 1 & 3.0892 & 0.6298 & 0.55672 & 1 & $1.64 \mathrm{E}-18$ & 0.0192 & 0.0645 \\
\hline$i_{5}$ & 0.8 & 2.6359 & 0.67195 & 0.56048 & 1 & 1.25E-24 & 0.0192 & 0.0746 \\
\hline$i_{5}$ & 0.6 & 2.9661 & 0.62938 & 0.55315 & 1 & 1.41E-09 & 0.0192 & 0.0458 \\
\hline is & 0.5 & 2.9836 & 0.63758 & 0.58806 & 1 & 1.78E-09 & 0.0192 & 0.0456 \\
\hline$i_{5}$ & 0.45 & 3.1455 & 0.62375 & 0.52021 & 1 & $1.82 \mathrm{E}-07$ & 0.0192 & 0.0402 \\
\hline$i_{5}$ & 0.4 & 3.4035 & 0.6429 & 0.56318 & 1 & 5.06E-04 & 0.0192 & 0.0287 \\
\hline$i_{5}$ & 0.3 & 3.7789 & 0.61718 & 0.51631 & 0 & 0.2839 & 0.0192 & 0.0139 \\
\hline i6 & 1 & 3.2594 & 0.61485 & 0.53234 & 1 & $6.40 \mathrm{E}-14$ & 0.0192 & 0.0557 \\
\hline$i_{6}$ & 0.8 & 3.088 & 0.64219 & 0.54102 & 1 & $5.51 \mathrm{E}-13$ & 0.0192 & 0.0537 \\
\hline i6 & 0.6 & 3.1714 & 0.64848 & 0.59233 & 1 & 1.50E-10 & 0.0192 & 0.0482 \\
\hline$i_{6}$ & 0.5 & 3.2267 & 0.65057 & 0.58373 & 1 & 9.70E-10 & 0.0192 & 0.0463 \\
\hline$i_{6}$ & 0.45 & 3.267 & 0.63234 & 0.56437 & 1 & 5.29E-07 & 0.0192 & 0.0389 \\
\hline i6 & 0.4 & 3.3155 & 0.6084 & 0.50981 & 1 & 3.59E-05 & 0.0192 & 0.033 \\
\hline$i_{6}$ & 0.3 & 3.4349 & 0.60951 & 0.51533 & 1 & 0.0028 & 0.0192 & 0.0256 \\
\hline
\end{tabular}

1.0, and the hypothesis is denied that the distributions of returns follow the corresponding Gaussian distribution. In Table 4 , when $\dot{i}=0.3$, only the returns with the plus boundary condition ${ }_{i 6}$ fail to follow the Gaussian distribution, which is different from the other five classes boundary conditions. 


\subsection{Power-law behavior and fractal phenomena of financial time series}

The aim of this part is to study the power law behavior of the financial model and the real stock markets by comparison. The tail probability distributions of market returns are found empirically to be (see [8])

$$
P(|r(t)|>x) \sim x^{-\alpha}
$$

for some $i \dot{ } i 3$. The main feature of this function is invariance of scale, in other words, the shape of the function is preserved. Power-law distributions show no typical scale or size, and in some cases they are connected with fractals, which also lack typical scales. Power-law distributions occur very often in natural and social fields. A few notable examples are Pareto's law for income distributions, behavior near a secondorder phase transition and Zipf's law. They are commonly cited as examples of power laws.

In Tables 3 and 4, they exhibit that the returns follow the power law distributions for the tails, the ordinary least square estimate yields $i=3.0 \pm 0.1$ for the three real stock market indexes, we refer to this phenomenon as "the cubic law of returns". In Table 3, the values of exponents $i$ are given for returns of SSE, SZSE, and Hushen 300. In Table 4 , according to the simulation of the financial time series, the value $i$ varies from 2.6359 to 3.7789 . Figure $4 \mathrm{a}, \mathrm{b}$ and Table 4 display that, for the fixed boundary condition $i_{4}$, the smallest value of $\dot{i}$ is 2.9488 , and the largest one is 3.7723 , the tail probability distributions of the simulated market returns are different. Similarly, we can discuss other cases for different boundary conditions and different parameters. In Figure 4c, $\mathrm{d}$ and Table 4 , with the fixed intensity parameter $\dot{c}=0.6$, the tail probability distributions change for different boundary conditions, and the tail distribution of $i 6$ in Figure 4c declined quicker than the others. In Figure 4e, f, the comparisons between the Chinese stock markets and the simulative data are given. The plot and the semilog plot of cumulative distributions of simulated returns for $\dot{i}=0.5$ and $\dot{i}=0.6$ with the boundary condition $i_{5}$, and the corresponding plots of returns for Hushen 300 and SSE are given in Figure 4e. The log-log plot and the semilog plot of cumulative distributions of simulated returns for $\dot{i}=0.6$ with zero boundary condition $\dot{i 1}$, and the corresponding plots of returns of SZSE are given in Figure 4f. These empirical results show that the price model is accord with the real market to some degree. In Table 4, the interval of the tails exponents of $i 6$ is [3.088,3.4349], which is much smaller than the range intervals of other five classes boundary conditions, which can show that the intensity parameter $\dot{i}$ has a relative weak effect on the tails distributions of financial model with boundary condition $i 6$ by comparison with other boundary conditions.

Mandelbrot [17] verified that the empirical relation discovered by Hurst exhibited the same form as the one presented by the series that describe the Brownian fractional movement, regarding the rescaled range $R / S$ in function to the period used in the calculus $N$ and, therefore, that the Hurst exponent $H$ could be used to represent long memory properties. The Hurst exponent $H$ is defined in terms of the asymptotic behavior of the rescaled range as a function of the time span for a time series as follows [18]:

$$
E\left[\frac{R(N)}{S(N)}\right]=C N^{H}, \quad N \rightarrow \infty
$$




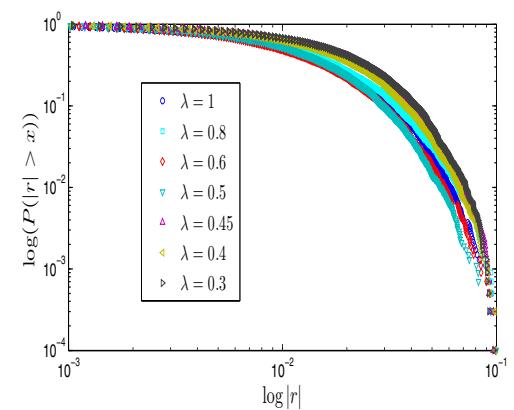

(a)



(c)

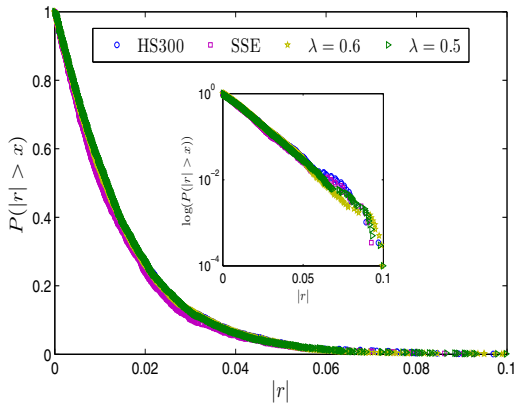

(e)

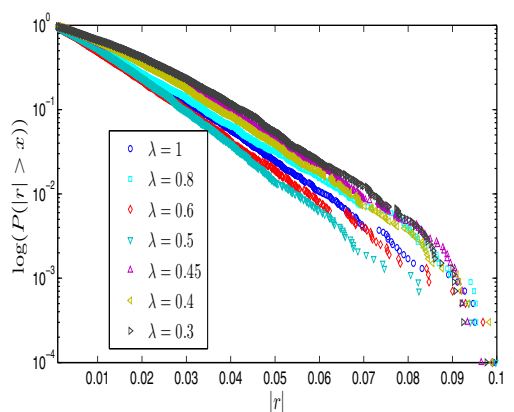

(b)

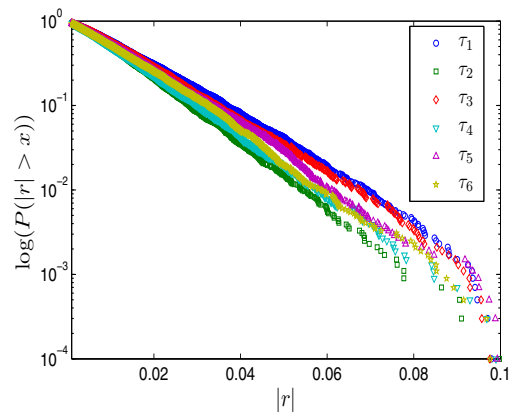

(d)



(f)

Figure 4 The plots of cumulative distributions of the stock returns. (a, b) The log-log plot and the semilog plot of cumulative distributions for the returns of the Ising-like financial model with the boundary condition of i4. (c, d) The log-log plot and the semilog plot of cumulative distributions for the returns of the Ising-like financial model with $i=0.6$. (e) The plot of cumulative distributions and the semilog plot of the simulated returns for $i=0.5$ and $i=0.6$ with the boundary condition $i_{5}$, and the returns of Hushen 300 and SSE. (f) The log-log plot of cumulative distributions of the simulated returns for $i=0.6$ with zero boundary condition $i_{1}$ and the returns of SZSE.

where $\left[\frac{R(N)}{S(N)}\right]$ is the rescaled range, $E[x]$ is the expected value, $N$ is the number of data points in a time series, $C$ is a constant.

Hurst exponent is referred to as the index of dependence, and is the relative tendency of a time series to either strongly regress to the mean or cluster in a direction. When $0 i_{i} H_{i} 0.5$, the analyzed series is anti-persistent, presenting reversion to the mean; if $H=0.5$, the series presents random walk; and if $0.5 i H_{i} 1$, the series is 
persistent, with the maintenance of tendency. This develops a method of the long memory estimates for the volatilities series. The long memory is measured by the Hurst exponent $H$, calculated through the rescaled range analysis $(R / S)$, which can be described as follows, for details, see [19].

Step 1: We define $i t$-returns as

$$
r(\Delta t)=\ln S(t)-\ln S(t-\Delta t), \quad \Delta t=1,2, \ldots, T .
$$

Thus, we obtain the new time series of $i t$-returns.

Step 2: Then we divide time series of $i t$-returns into $s$ subseries with length $q$ :

$$
E_{q, k}(\Delta t)=\left\{r_{1, k}(\Delta t), r_{2, k}(\Delta t), \ldots, r_{q, k}(\Delta t)\right\}, \quad k=1,2, \ldots, s
$$

where $q=[N / s]$ and $N$ is the number of observation.

Step 3: From the time series of $i t$-returns, the deviation $D_{q, k}(i t)$ can be defined directly from the mean of returns $\bar{r}_{q, k}(\Delta t)$ as

$$
D_{q, k}(\Delta t)=\sum_{d=1}^{q}\left(r_{d, k}(\Delta t)-\bar{r}_{q, k}(\Delta t)\right), \quad k=1,2, \ldots, s
$$

where

$$
R_{q, k}(\Delta t)=\max \left\{D_{i, k}(\Delta t)\right\}-\min \left\{D_{i, k}(\Delta t)\right\}, \quad i=1, \ldots, q .
$$

Step 4: Thus, the hierarchical average value $(R / S)_{N}(i t)$ that stands for the relation between $R_{N, k}(i t)$ and $S_{q, k}(i t)$ becomes

$$
(R / S)_{q}(\Delta t)=\frac{1}{s} \sum_{k=1}^{s} \frac{R_{q, k}(\Delta t)}{S_{q, k}(\Delta t)} \propto q^{H(\Delta t)}
$$

where $H\left({ }_{i} t\right)$ stands for Hurst exponent and

$$
S_{q, k}(\Delta t)=\sqrt{\frac{1}{q} \sum_{d=1}^{q}\left(r_{d, k}(\Delta t)-\bar{r}_{q, k}(\Delta t)\right)}, \quad k=1,2, \ldots, s
$$

Step 5: Hurst exponents can be obtained by linear regression using

$$
\ln (R / S)_{q}=\ln (c)+H(\Delta t) \cdot \ln (q) .
$$

The main purpose of inducing and computing $V$ statistics is to find the nonperiodic cycles by observing relative map of that statistics, in that if the curve of that statistics is horizontal, the time series under study is random one which follows random walks, otherwise, there exists long-term memory in the time series. If there are any critical points, the $q$ in these points stands for the length of the nonperiodic cycles, that is, the memory of system information will be lost in the system after $q$ days. $V$ statistics can be defined as

$$
V_{q}=\frac{(R / S)_{q}}{\sqrt{q}}
$$

In Figure 5, we concentrate on the fractal analysis of absolute return time series of SSE Index and the corresponding simulative data of the financial model with boundary 




(a)

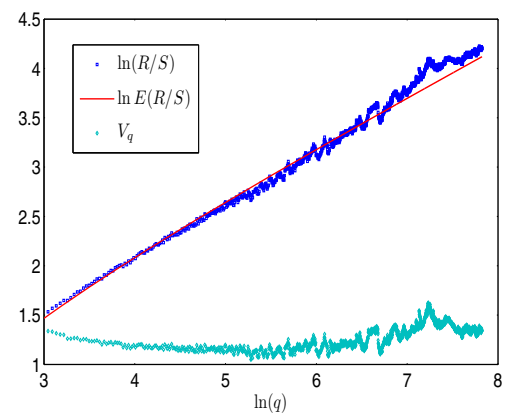

(c)

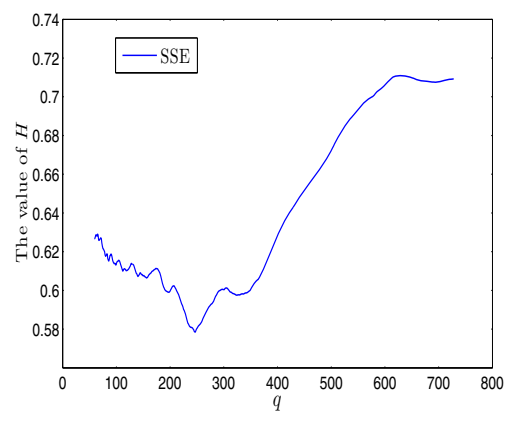

(b)

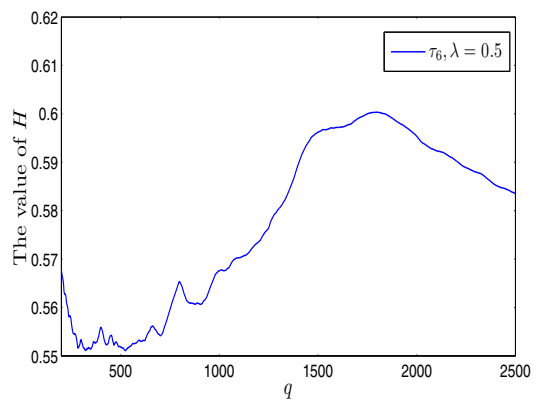

(d)

Figure 5 The fractal analysis of absolute return time series for SSE index and the financial model. $(\mathbf{a}, \mathbf{c})$ Volatility term structure of the absolute simulative returns with $i \sigma, i=0.5$ and SSE Index returns respectively. (b, d) The values of $H$ depend on different blocks $n$ of absolute simulative returns with $i \sigma_{1} i=$ 0.5 and SSE Index returns respectively.

condition $i_{6}$ and $i=0.5$ by using $R / S$ analysis with $i t=1$. From Figure $5 \mathrm{a}, \mathrm{c}$ we can obtain that the volatility exists both in the real stock market and the simulative data, but the volatility in the simulative time series is a little weaker than that in the SSE Index absolute returns. We also obtain the relation between the exponent $H$ and $n$. The way we calculate is that $n=60$ is the starting point of the regression; gradually increasing $n$ to obtain a value of $H$ regressing once for each additional day, up to $50 \%$ of the length of the sequence. We also find in Figure 5b, d that the values of $H$ are all larger than 0.5 for SSE Composite Index and the data of simulation.

Next, we change the procedure of step 2 as follows: we divide time series of $i t$ returns into $s^{\prime}=\left[\log _{2}(N)\right]$ subseries with length $q^{\prime}$ :

$$
E_{q^{\prime}, k^{\prime}}(\Delta t)=\left\{r_{1, k^{\prime}}(\Delta t), r_{2, k^{\prime}}(\Delta t), \ldots, r_{q^{\prime}, k^{\prime}}(\Delta t)\right\}, \quad k^{\prime}=2^{0}, 2^{1}, \ldots, 2^{s^{\prime}} .
$$

where $q^{\prime}=2^{s^{\prime}} / 2^{k^{\prime}}$ and $N$ is the number of observation. And we also change the step 5 to be

$$
\log _{2}(R / S)_{q^{\prime}}=\log _{2}(c)+H(\Delta t) \cdot \log _{2}\left(q^{\prime}\right) .
$$

Then, we can get the Hurst parameter $H_{1}$ from regression on all data of $\log _{2}(R / S)_{q^{\prime}}$ and the Hurst parameter $\mathrm{H}_{2}$ from regression on means of $\log _{2}(R / S)_{q^{\prime}}$ for each segmentation of length $q$ ' of the time series, see in Tables 3, 4 and Figure 6. From Table 4, for 




(a)



(b)

Figure 6 The plots of the Hurst parameters from regression analysis. (a) The Hurst parameter from regression on all data and the Hurst parameter from regression on segmentation means for Hushen 300 Index. (b) The Hurst parameter from regression on all data and the Hurst parameter from regression on segmentation means for the simulated data with $i_{6}, i=0.5$.

most financial time series except the boundary conditions $\dot{i 1}, \dot{i} 4$ with $\dot{i}=0.45$ and $i 3$ with $\dot{i}=0.45, \dot{i}=0.3$, the Hurst parameter $H_{1}$ and $H_{2}$ are larger than 0.5. In Figure 6, $H>0.5$ for returns of Hushen 300 Index and the data of simulation. The above analysis of fractal behaviors show that the long-memory exists in returns, and these time series are persistent with the maintenance of tendency.

\section{Conclusion}

For the financial modeling, any model aiming at understanding price fluctuations needs to define a mechanism for the formation of the price. In the present article, the financial model based on the Ising-like spin system is the contributor towards our ultimate understanding of the impact of external condition and interaction among the market investors and critical phenomena of the empirical stock markets. In this financial model, we suppose that the financial market not only depends on the perspective that the price movements are caused primarily by the external environment, but also depends on the spread of investing information which is due to the interaction among the market investors. The boundary condition $\dot{i}$ may represent the information or the situation on this stock, including the estimate for this stock price, positive or negative news, trends, political event and economic policy, etc. The parameter $i$, phase transitions and critical phenomena of the model can be explained as the intensity of interaction among the market participants in the financial market.

In the model, the intensity parameter $i$ represents the strength of information spread and the depth parameter $i$ describes the strength of market fluctuation, both of them are defined in Section 3. Note that the range of daily price fluctuation is limited in Chinese stock markets, that is, the changing limits of daily returns for stock prices and stock market indexes are between $-10 \%$ and $10 \%$. In order to make the financial price model satisfy the changing limits of daily returns for Chinese stock markets, the value of $i$ is chosen dependently on the value of $i$ in Section 4. According to the empirical research of the model in Table 2, for each fixed boundary condition $\dot{i}$, the value of depth parameter $i$ has the tend to decrease as the intensity parameter $i$ is increasing. And for each fixed value of intensity parameter $\dot{i}$, when the boundary conditions are neither " + " nor " - " predominates the other, for example the boundary conditions $\dot{\iota}_{1}$, 
$\dot{i}_{2}$ and $i_{3}$ (which are usually called the "weak boundary conditions"), the corresponding values of depth parameters $i$ are larger than that of depth parameter $\dot{i}$ of plus boundary condition $i 6$ (when the plus (or the minus) is the overwhelming part of boundary sites, it is usually called the "strong boundary condition"). The large value of intensity parameter $\dot{i}$ of the financial price model will exhibit the strong interaction among the market participants, this implies that the information or news about the market may spread far and wide among the market investors. The strong boundary condition of the model also shows that the external investing environment has a strong impact on the fluctuation of financial market. In the present article, by following the trading rules of Chinese stock markets, we find that when the interaction among the market investors increases or the external environment is dominant by one view for a long time, the value of depth parameter $i$ may decrease. This behavior may suggest that the possibility of long time continuous large volatilities of stock prices is small.

In Tables 3 and 4, the tails power law distributions and the fractal behaviors of market returns for the price model and the real stock markets are analyzed by empirical research and computer simulation. The values of exponents $\dot{i}$ of returns are around the value 3 for the financial indexes of SSE, SZSE and Hushen 300. For the boundary condition $i_{6}$ of the model, the largest value and the smallest value of tails exponent $i$ are 3.4349 and 3.088, respectively. Then the changing range of tails exponents of boundary condition $i 6$ is 0.3469 (= $3.4349-3.088)$, which is much smaller than the corresponding changing ranges of tails exponents of other five boundary conditions. This shows that the intensity $\dot{i}$ of the model with the boundary condition ${ }_{i} 6$ has a weak impact on the tails distributions by comparing with other boundary conditions cases. This behavior is due to that the strong boundary conditions (for example the boundary condition $i 6$ ) or the strong external environment have a deep influence on the price dynamics in this work. According to the evolution of the price model which is modelled by Ising-like dynamic system, the strong external environment may make most of market participants take a similar investing strategy. So that, for the boundary condition $i 6$, the interaction among the market participants has a relative weak effect on the price fluctuation, and the tails distributions exhibit more stable behavior for different values of intensity $i$ (by comparison with other boundary conditions cases).

In Section 4, the empirical analysis displays that the fractal behaviors and the persistence properties exist in the real Chinese stock markets, SSE, SZSE, and Hushen 300. And we also analyze the fractal behaviors and other statistical properties of the financial model with six kinds of boundary conditions, and we also investigate the fluctuations of exponents $H$ of absolute returns for the real markets and the price model. The empirical research shows the price financial model also exhibits fractal and persistence properties for different boundary conditions, this means that the six kinds of boundary conditions of this article can not change the existence of fractal behaviors for the absolute returns of the model. From the above summary, we think that the financial model of the present article is reasonable for the real stock market to some extent.

\section{Acknowledgements}

The authors were supported in part by National Natural Science Foundation of China Grant No. 70771006 and Grant No. 10971010, Fundamental Research Funds for the Central Universities No. 2011YJS077, and BJTU Foundation No. S11M00010. The authors would like to thank the support of Institute of Financial Mathematics and Financial Engineering in BJTU. Thanks to the anonymous referees for their useful comments and suggestions, which helped us to improve our work. 


\section{Authors' contributions}

We are part of the same research group and work together therefore, we can affirm that the contents of this article has been prepared by all the authors: WF and JW. All authors read and approved the final manuscript.

\section{Competing interests}

The authors declare that they have no competing interests.

Received: 20 May 2011 Accepted: 2 February 2012 Published: 2 February 2012

\section{References}

1. Krawiecki, A: Microscopic spin model for the stock market with attractor bubbling and heterogeneous agents. Int J Modern Phys C. 16, 549-559 (2005). doi:10.1142/S0129183105007285

2. Zhang, JH, Wang, J: Modeling and simulation of the market fluctuations by the finite range contact systems. Simulat Model Pract Theor. 18, 910-925 (2010). doi:10.1016/j.simpat.2010.02.008

3. Wang, J, Wang, QY, Shao, JG: Fluctuations of stock price model by statistical physics systems. Math Comput Model. 51, 431-440 (2010). doi:10.1016/j.mcm.2009.12.003

4. Wang, J: The estimates of correlations in two-dimensional Ising model. Physica A: Stat Mech Appl. 388, 565-573 (2009). doi:10.1016/.jphysa.2008.11.016

5. Lamberton, D, Lapeyre, B: Introduction to Stochastic Calculus Applied to Finance. Chapman and Hall/CRC, London (2000)

6. Bouchaud, JP, Potters, M: Theory of Financial Risk and Derivative Pricing: From Statistical Physics to Risk Management. Cambridge Univ. Press, Cambridge (2003)

7. Johnson, NF, Jefferies, P, Hui, PM: Financial Market Complexity. Oxford Univ. Press, Oxford (2003)

8. Lux, T: The stable paretian hypothesis and the frequency of large returns: An examination of major German stocks. Appl Financial Econ. 6, 463-475 (1996). doi:10.1080/096031096333917

9. Anderson, PW: More is different. Science. 177, 393-396 (1972). doi:10.1126/science.177.4047.393

10. Chen, MF: From Markov Chains to Non-Equilibrium Particle Systems. World Scientific, Singapore (1992)

11. Durrett, R: Lecture Notes on Particle Systems and Percolation. Wadsworth \& Brooks, Pacific Grove, CA (1998)

12. Liggett, T: Interacting Particle Systems. Springer, New York (1985)

13. Gaylord, R, Wellin, P: Computer Simulations with Mathematica: Explorations in the Physical, Biological and Social Science. Springer, New York (1995)

14. Ellis, RS: Entropy, Large Deviations, and Statistical Mechanics. Springer, New York271 (1985) Grundlehren der Mathematischen Wissenschaften

15. Wang, J: The Stochastic Ising Model with the Mixed Boundary Conditions. Boundary Value Problems. 2009, 1-18 (2009)

16. Marsaglia, G, Tsang, WW, Wang, J: Evaluating Kolmogorov's Distribution. J Stat Softw. 8, 1-4 (2003)

17. Mandelbrot, B: Statistical methodology for non-periodic cycles: from the covariance to R/S analysis. Ann Econ Soc Measur. 1, 259-290 (1972)

18. Feder, J: Fractals (Physics of solids and liquids). Plenum Press, New York (1988)

19. Peters, EE: Chaos and Order in Capital Markets: A New View of Cycles, Prices and Market Volatility. John Wiley and Sons, New York, 2 (1996)

doi:10.1186/1687-2770-2012-9

Cite this article as: Fang and Wang: Effect of boundary conditions on stochastic Ising-like financial market price model. Boundary Value Problems 2012 2012:9.

\section{Submit your manuscript to a SpringerOpen ${ }^{\circ}$ journal and benefit from:}

Convenient online submission

Rigorous peer review

- Immediate publication on acceptance

- Open access: articles freely available online

- High visibility within the field

- Retaining the copyright to your article

Submit your next manuscript at $\boldsymbol{s p r i n g e r o p e n . c o m ~}$ 